\title{
Reflets
}

Revue ontaroise d'intervention sociale et communautaire

\section{Le Regroupement des jeunes filles francophones : Oeuvrer pour l'épanouissement de la jeune fille francophone de Toronto}

\author{
Jane Turrittin
}

Volume 6, numéro 2, automne 2000

Problèmes sociaux en Ontario français

URI : https://id.erudit.org/iderudit/026328ar

DOI : https://doi.org/10.7202/026328ar

Aller au sommaire du numéro

Éditeur(s)

Reflets : Revue ontaroise d'intervention sociale et communautaire

ISSN

1203-4576 (imprimé)

1712-8498 (numérique)

Découvrir la revue

Citer cet article

Turrittin, J. (2000). Le Regroupement des jeunes filles francophones : Oeuvrer pour l'épanouissement de la jeune fille francophone de Toronto. Reflets, 6(2), 235-240. https://doi.org/10.7202/026328ar

Tous droits réservés (C) Reflets : Revue ontaroise d'intervention sociale et communautaire, 2000
Ce document est protégé par la loi sur le droit d'auteur. L'utilisation des services d'Érudit (y compris la reproduction) est assujettie à sa politique d'utilisation que vous pouvez consulter en ligne.

https://apropos.erudit.org/fr/usagers/politique-dutilisation/ 


\section{Le Regroupement des jeunes filles francophones de Toronto $\square$ :}

\section{Oeuvrer pour l'épanouissement de la jeune fille francophone de Toronto}

\section{Jane Turrittin}

Le Regroupement des jeunes filles francophones de Toronto existe depuis 1993 sous la houlette de Mademoiselle Léonie Tchatat. Plusieurs facteurs ont justifié la création de cet organisme, reconnu aujourd'hui d'intérêt public à Toronto.

Le premier facteur est, sans contredit, la nécessité de répondre aux besoins grandissants des jeunes filles. La pauvreté, l'emploi, l'intégration, la solidarité, les conflits familiaux, l'encadrement des jeunes sont les principaux du Regroupement, dont le travail est le fruit du courage, de la ténacité et de l'imagination d'une équipe soudée et dynamique gravitant autour de Mademoiselle Tchatat.

Pour l'année 1999-2000, le bilan est particulièrement flatteur comme en témoignent les faits qui suivent.

Parmi les activités du Regroupement, mentionnons le programme d'assistance aux jeunes filles nouvellement arrivées à Toronto et le projet de développement d'entreprise à vocation internationale qui a permis à des jeunes Canadiennes de faire un voyage au Cameroun afin d'apporter leur savoirfaire aux jeunes de cette contrée africaine.

Il faut souligner, par ailleurs, que "les groupes de discussions culturels ", la formation en coopérative des jeunes filles et les journées portes ouvertes ont également suscité un grand intérêt. 
Un forum communautaire, initié par le Regroupement le 15 mai 1999 avait pour thème : "La prostitution... si on en parlait ". Il a connu un véritable succès et un rapport en a été publié.Vous pouvez vous le procurer pour la modique somme de $10 \$$.

Avec l'imagination qui le caractérise, l'organisme a initié, en collaboration avec Oasis Centre des Femmes, un souper interculturel qui a attiré près de 70 personnes. Cette rencontre a connu un succès retentissant. Les fonds recueillis ont contribué à l'organisation du Forum 2000.

Intitulé "Prends soin de toi, prends soin de ton avenir ", ce forum provincial a rassemblé plus de 400 jeunes filles en provenance de tous les coins de la province. Au cours de cette rencontre, elles ont fait ressortir leurs besoins réels, sous forme de recommandations qui seront remises aux différentes instances gouvernementales et aux organismes communautaires.

Signalons la sortie prochaine d'un magazine pour jeunes filles francophones ethnoculturelles intitulé TALOUA.

Le Regroupement des jeunes filles francophones de Toronto n'a pas fini de nous étonner, car il constitue un foisonnement d'idées concrètes et pratiques!

Pour contacter le Regroupement des jeunes filles francophones de Toronto :

Téléphone : (416) 9340588

Télécopieur : (416) 9340590

Courriel :_ filles@istar.ca

Adresse postale : $\quad 22$, rue Collège, bureau 207

Toronto (Ontario) M5G 1K3

\section{Rapport des activités 1999-2000 du RJFFT}

\section{Forum communautaire du 15 mai $\square \square$ :}

La prostitution... si on en parlait.

Ce forum réunissait plusieurs membres de la communauté qui 
ont participé à différents ateliers et écouté les témoignages des panélistes invitées. Le rapport d'analyse du projet de rechercheaction intitulé : "La prostitution si on en parlait a été présenté ». Le document est disponible pour la somme de $10 \$$.

\section{Souper interculturel—souper de levée de fonds.}

Plus de 70 personnes ont assisté à ce souper interculturel organisé par le RJFFT, en partenariat avec Oasis pour le Forum 2000 : "Prends soin de toi, prends soin de ton avenir ».

\section{Programme de bénévolat}

Le RJFFT accueille les bénévoles de tous âges pour aider à accomplir ses projets. Leur dévouement est grandement apprécié. Une trentaine de bénévoles ont pu acquérir une expérience canadienne cette année.

\section{Programme d'assistance aux jeunes filles nouvellement arrivées}

Les jeunes filles viennent nous voir pour une multitude de raisons; on les accompagne dans des démarches soit médicales, de recherche d'emploi, d'intégration, d'orientation scolaire, d'immigration, dans des situations de violence, de conflit familial, etc.

\section{Ikuen-cuisine communautaire francophone interculturelle}

Une fois par mois, les jeunes filles francophones (défavorisées) participent aux sessions de formation culinaire interculturelle. Ces sessions sont coordonnées et animées par les jeunes filles; à tour de rôle, chacune présente une recette de son pays d'origine.

\section{Livret de recettes interculturelles}

Les jeunes filles ont aussi travaillé très fort sur la conception d'un livret de recettes qui comprend une série de recettes qu'elles ont choisies. Le livret sera disponible dès 2001.

\section{Prévenir vaut mieux que guérir}

Des sessions d'information en auto-défense ont été offertes aux jeunes filles dans le but de les informer sur leur environnement 
immédiat afin de mieux les prévenir face à des situations désagréables de violence auxquelles elles pourraient être confrontées.

\section{Projet de développement d'entreprise à vocation internationale}

En novembre 1999, une délégation de 7 jeunes filles canadiennes est partie au Cameroun pour une période de 6 semaines. Ces dernières ont pu développer, sur le terrain, leurs aptitudes dans une entreprise dans leur domaine d'expertise, et en échange, apporter leur expérience canadienne aux entreprises camerounaises.

\section{Ligne 1-888-367-8620}

Une ligne qui permet à toutes les jeunes filles du Canada de nous rejoindre et obtenir les renseignements dont elles ont besoin.

\section{Un site Web}

Le site $\boldsymbol{w} \boldsymbol{w} \boldsymbol{w}$.jeunesfilles.com est rempli d'informations et de détails sur tous les projets qui ont été accomplis au fil des années.

\section{Taloua-magazine pour jeunes filles francophones ethnoculturelles}

Magazine pour les jeunes femmes modernes et dynamiques, âgées de 18 à 35 ans, qui vont exprimer leurs réalités, leurs aspirations, ceci dans un contexte multiculturel, et à partir de cela, s'ouvrir sur le monde.

\section{Comité consultatif pour la conférence sur l'anti- racisme qui aura lieu en Afrique du Sud}

Une première rencontre de consultation s'est tenue à Ottawa avec la Ministre Hedy Fry à propos de la position du Canada en ce qui concerne la lutte anti-racisme. Le comité s'est ensuite réuni à Toronto, les 25 et 27 septembre 2000 pour déterminer les procédures pour la conférence sur l'anti-racisme qui aura lieu en Afrique du Sud en 2001.

\section{Forum 2000 $\square$ : Prends soin de toi, prends soin de ton avenir}

Le premier forum provincial pour les jeunes filles francophones 
dans toute leur diversité a rassemblé plus de 400 jeunes filles en provenance de tous les coins de l'Ontario. Ces jeunes filles ont pu s'exprimer ouvertement sur les réalités qu'elles vivent au tournant du millénaire, se réseauter et prendre connaissance des services et organismes qui desservent les francophones. Les jeunes filles ont fait ressortir leurs besoins réels sous forme de recommandations qui seront remises aux différentes instances gouvernementales et aux organismes communautaires.

\section{Foire aux organismes}

Vingt-deux organismes francophones se sont réunis sous le toit du Trading Floor au prestigieux Design Exchange pour faire connaitre leurs services aux jeunes filles lors du Forum 2000 à Toronto. Celles-ci se sont réjouies de cette foire d'information.

\section{Soirée cocktail}

Une soirée pour souligner l'importance de la tenue du Forum 2000 à travers la province, agrémentée de discours poignants, de danses, de chants, de témoignages, etc.

\section{Plan stratégique}

Le RJFFT se penche maintenant sur l'élaboration d'un plan stratégique qui pourra répondre aux recommandations prioritaires qui sont ressorties du Forum 2000 à Toronto.

\section{Formation en coopérative}

Les jeunes filles du RJFFT ont suivi une formation pour leur permettre de mieux s'orienter dans le développement d'une coopérative. Elles continueront à suivre une série d'ateliers pour approfondir leur connaissance et leur aptitude en entrepreunariat.

\section{LOGICIEL : Se lancer en affaires c'est facile, mais y demeurer c'est difficile!}

Un logiciel pour aider les jeunes filles qui veulent se lancer en affaires. 


\section{Portes ouvertes}

Une occasion pour les jeunes filles de venir visiter nos bureaux, prendre de l'information et socialiser.

\section{Groupes de discussion culturels}

Favoriser les échanges entre les jeunes filles de la communauté ethnoculturelle sur différent thèmes d'ateliers, avec une approche plutôt culturelle et traditionnelle.

\section{Journée de reconnaissance}

Reconnaitre les personnes qui se sont investies pleinement durant l'année et les bénévoles du Regroupement qui ont donné de leur temps.

\section{Place du Canada}

Huit jeunes ont participé à une activité de chasse au trésor sur l'internet à la Place du Canada, et trois des participants ont remporté des prix dont un ordinateur et une caméra numérique.

\section{Groupe d'échange France-Canada}

Le RJFFT a reçu plusieurs groupes de jeunes arrivant de la France dans le cadre de leur projet « Passeport Monde 2000 ». Les jeunes ont pu échanger sur leur propre culture, sur les différences entre les deux pays, sur leurs intérêts, etc. Ils ont même échangé leur adresse pour garder contact avec la jeunesse canadienne.

Pour plus d'informations, composez le (416) 934-0588

ou encore le1-888-FORUM2000

ou visitez notre site Web www.jeunesfilles.com. 